\title{
SUPPRESSION OF CAVITATION INSTABILITIES IN AN INDUCER BY J-GROOVE
}

\author{
Noriyuki SHIMIYA, Osaka University, Osaka, Japan. \\ Akira FUJII, Osaka University, Osaka, Japan. \\ Hironori HORIGUCHI, Osaka University, Osaka, Japan. \\ Masaharu UCHIUMI, JAXA, Sendai, Japan. \\ Junichi KUROKAWA, Yokohama National University, Yokohama, Japan. \\ Yoshinobu TSUJIMOTO, Osaka University, Osaka, Japan.
}

\begin{abstract}
The suppression of cavitation instabilities was attempted through the control of tip leakage vortex cavitation. The control was made by using shallow grooves, called J-groove, on the casing wall. With Jgroove, the onset regions of the rotating cavitation and the asymmetric cavitation could be diminished. However, a cavitation surge appeared at higher cavitation numbers. From the observation of cavitation, it was found that the cavitation surge occurred when the tip leakage vortex cavitation started to interact with the leading edge of the next blade. This type of cavitation surge could be avoided by extending the leading edge of J-groove upstream. However, in this case, another type of cavitation surge occurred at much lower cavitation numbers, which was caused by the cavitation between the blade surface and the tip leakage vortex cavitation. These results highlight the importance of the tip leakage vortex cavitation for cavitation instabilities.
\end{abstract}

\section{INTRODUCTION}

Cavitation instabilities such as the rotating cavitation and the cavitation surge occur in turbopump inducers for rocket engines and cause shaft vibrations and blade stress fluctuations. One of the effective methods for the suppression of the cavitation instabilities is to expand the casing diameter upstream of the inducer inlet (Kamijo, K. et. al., 1993). However, there are certain cases in which cavitation instabilities cannot be suppressed perfectly. So, alternative methods for the suppression of cavitation instabilities are needed to realize reliable turbopumps.

Our research group tried to suppress the cavitation instabilities by shallow grooves, called J-groove (Imamura, H., et. al., 2003), on the casing wall near the inducer inlet for the development of a new method for the suppression of the cavitation instabilities (Fujii, A., et. al., submitted). In an inducer with larger blade angle, the cavitation surge was suppressed almost completely (Kurokawa, J., et. al., 2005). In an inducer with smaller blade angle, the rotating cavitation was suppressed by Jgroove, but the cavitation surge occurred at higher cavitation numbers (Fujii, A., et. al., submitted).
The present paper reports about further attempts to suppress cavitation instabilities by $\mathrm{J}$-groove and discusses about the correlation between tip leakage cavitation and cavitation instabilities.

\section{NOMENCLATURES}

$D$ :Diameter of inducer

$f \quad$ :Frequency of fluctuation

$f_{n} \quad$ Frequency of shaft rotation

$L \quad$ :Axial length of J-groove

$L_{1}$ :Axial length of J-groove upstream of the leading edge of inducer

$L_{2}$ :Axial length of $\mathrm{J}$-groove downstream of the leading edge of inducer

$p_{1}$ :Inlet pressure

$p_{1 t}$ :Inlet total pressure

$p_{2}$ :Outlet pressure

$p_{v}$ :Saturated vapor pressure

$\Delta p$ :Pressure fluctuation

$r \quad$ :Radial position

$R \quad$ :Radius of inducer

$U_{t}:$ Tip speed, $=\pi D f_{n}$

$u_{z}:$ :Axial velocity

$u_{\theta}$ :Tangential velocity

$v_{1}$ :Mean axial velocity at inducer inlet, $=$ flow rate $/$ inlet area

$z$ :Axial position measured downstream from tip leading edge

$\beta:\left(\beta_{t 1}\right.$ :Inlet blade angle at tip, $\beta_{2}$ :Outlet blade angle at tip)

$\rho \quad$ :Density

$\sigma \quad$ :Cavitation number, $=\left(p_{1}-p_{v}\right) /\left(\rho U_{t}^{2} / 2\right)$

$\phi \quad$ :Flow coefficient, $=v_{1} / U_{t}$

$\phi_{d}$ :Design flow coefficient

$\psi_{s}$ :Static pressure coefficient, $=\left(p_{2}-p_{1}\right) /\left(\rho U_{t}^{2}\right)$

$\Delta \psi:$ Fluctuating pressure coefficient, $=\Delta p /\left(\omega U_{t}^{2}\right)$

\section{EXPERIMENTAL FACILITY}

Figure 1 shows the cavitation tunnel used in the present study. This tunnel is a closed-loop type tunnel, and the cavitation number is adjusted by changing the pressure of the air in the upper part of a tank by a vacuum pump. Working fluid is water, and experiments are 


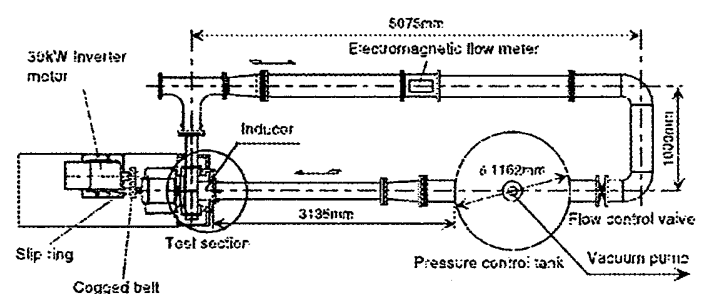

(a) Top view

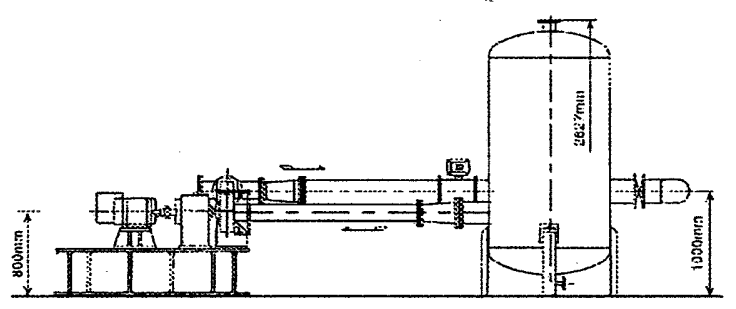

(b) Side view

Fig.1 Test facility
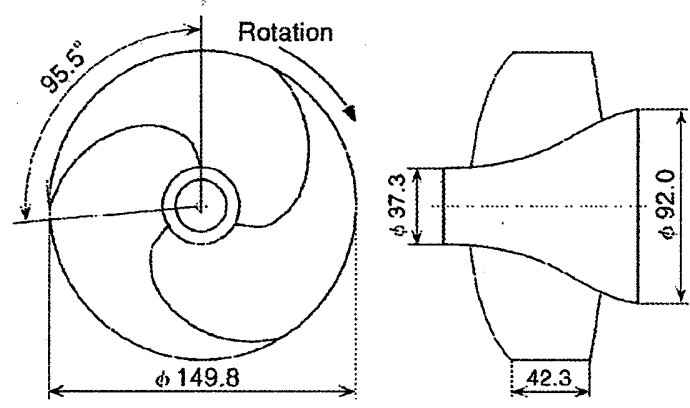

units in $\mathrm{mm}$
Fig.2 Șketch of test inducer

performed after the deaeration of water. Figure 2 shows the sketch of a test inducer, and Table 1 shows principal dimensions.

Pressure fluctuation was measured at the inlet of inducer by means of 4 pressure transducers flush mounted on the casing wall as shown in Fig.3, placed at intervals of 90 degrees circumferentially. The type of cavitation instability was determined from the phase difference of the signals from these pressure transducers. Casings were made of clear acrylic resin for visual observation and L.D.V. measurements. The rotational speed was $3000 \mathrm{rpm}$ $(50 \mathrm{~Hz})$.

\section{RESULTS AND DISCUSSIONS}

Figure 3 shows a cross-section of the inducer and $\mathrm{J}$ groove. The shape and arrangement of J-groove were decided in consideration of the previous research (Kurokawa, J., et. al., 2005). Axial length of J-groove upstream and downstream from the leading edge of the inducer, $L_{1}$ and $L_{2}$, are both $20 \mathrm{~mm}$. The width of the groove is $10 \mathrm{~mm}$ and the depth is $3 \mathrm{~mm}$. The number of the
Table 1 Principal dimensions of test inducer

\begin{tabular}{|l|c|}
\hline Number of blades & 3 \\
Tip dianeter, $\mathrm{D}(\mathrm{mm})$ & 149.8 \\
Inlet blade angle at tip, $\beta_{t 1}$ & $7.5 \mathrm{deg}$. \\
Outlet blade angle at tip, $\beta_{t 2}$ & $9.0 \mathrm{deg}$ \\
Hub / tip ratio at inlet & 0.25 \\
Hub $/$ tip ratio at outlet & 0.51 \\
Solidity at tip & 1.91 \\
Design flow coefficient, $\phi_{d 1}$ & 0.078 \\
\hline
\end{tabular}

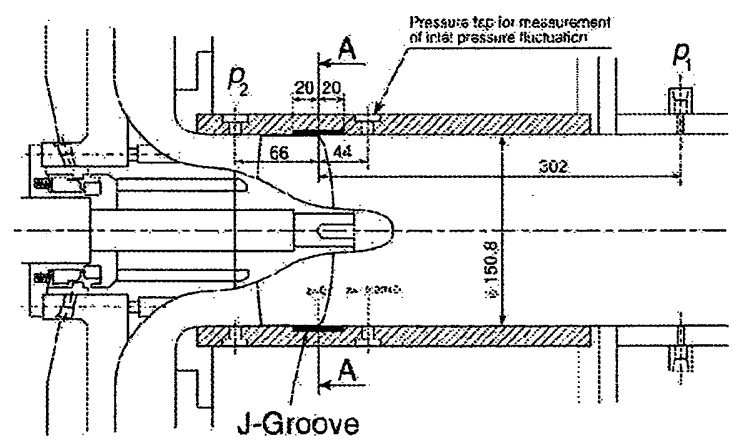

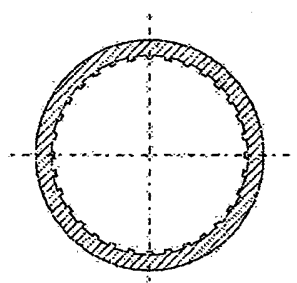

A-A section

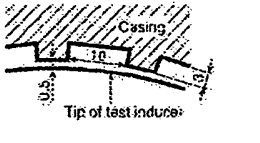

Units in $\mathrm{mm}$
Fig. 3 Cross-section of the inducer and J-groove

grooves on the casing wall is 32 . This groove is called JG1 $(20,20)$ in the present study. Figure 4 shows the occurrence regions of cavitation instabilities on the suction performance curves, without and with $\operatorname{JG} 1(20,20)$ (Fujii, A., et. al., submitted). In the case with JG1 $(20,20)$, the rotating cavitation (R.C.) and the asymmetric cavitation (A.C.) observed without the groove were almost suppressed. However, the cavitation surge (C.S.) appeared at higher cavitation numbers.

Velocity distributions at the inducer inlet were obtained by L.D.V.. About 1000 data were averaged at each measurement point. Figures 5(a), (b) show axial and tangential velocity distributions at the design flow coefficient: measured at $z / D=-0.294$. The data in the case without J-groove were obtained by a pitot tube with 3 holes by Maekawa et al. (Maekawa, Y., 1996). With Jgroove, backflow and prerotation in the vicinity of the casing wall disappear. This shows that J-groove can suppress prerotation and backflow in the upstream. 


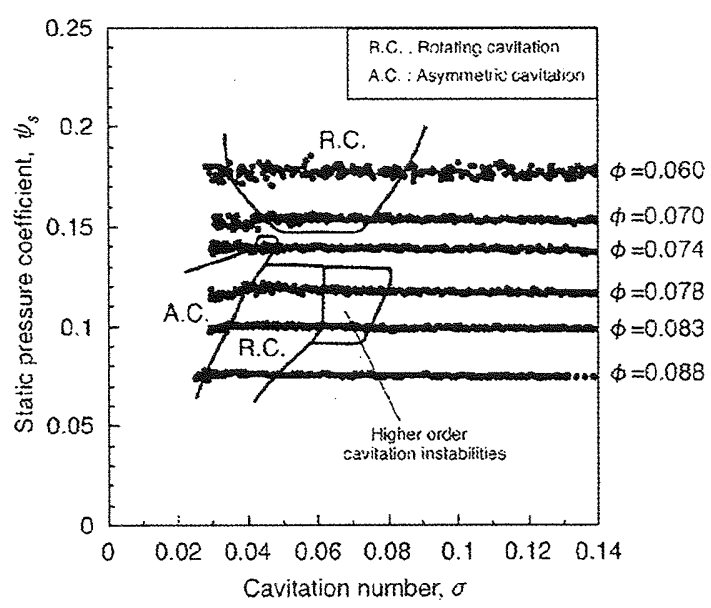

(a) Without J-groove

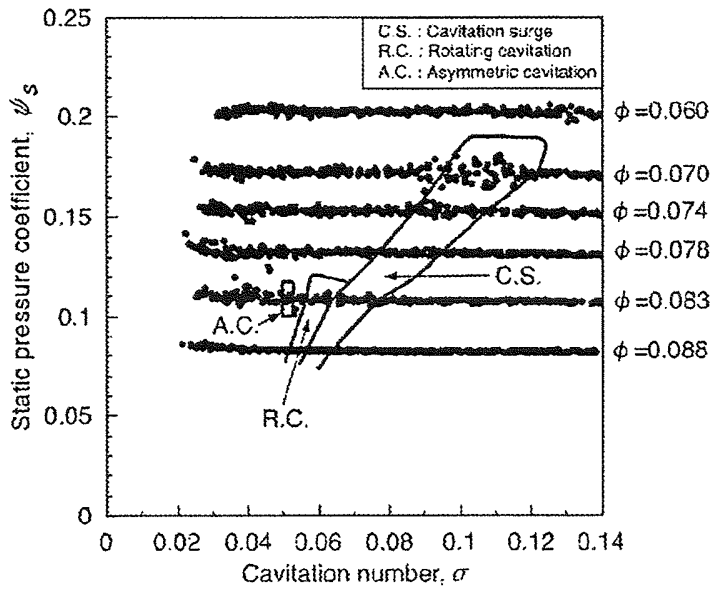

(b) With JG1 $(20,20)$

Fig.4 The occurrence regions of cavitation instabilities on the suction performance curves at various flow coefficients. Rotational speed is $3000 \mathrm{rpm}$.

Figure 6 shows the cavities at the design flow coefficient $\phi=0.078$ and the cavitation number $\sigma=0.120$, with and without JG1 $(20,20)$. With J-groove, the tip cavitation rolls up into a vortex and detaches from the blade surface. The tip leakage vortex cavitation bends to circumferential direction near the leading edge of $\mathrm{J}$ groove, and extends towards the leading edge of the next blade. With J-groove, the cavity length is larger than that without J-groove. When the cavitation number decreases, the tip vortex cavitation becomes longer, and the cavitation surge starts to occur at the cavitation number where the tip leakage vortex cavitation starts to interact with the leading edge of next blade (Fujii, A., et. al., submitted). This suggests that the cavitation surge at the higher cavitation numbers with $J G l(20,20)$ is caused by the interaction. Since the tip leakage vortex cavitation is bent to circumferential direction near the leading edge of the grooves, it is expected that the interaction and hence

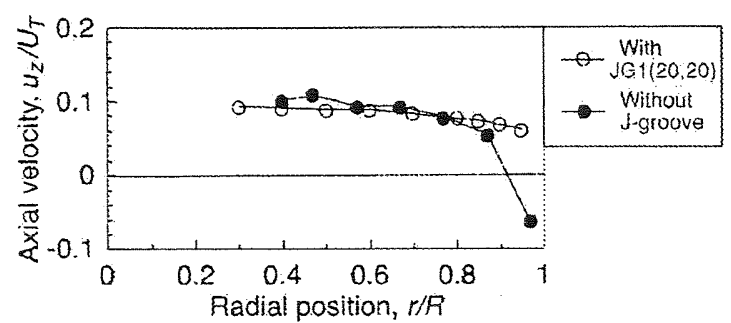

(a) Axial velocity

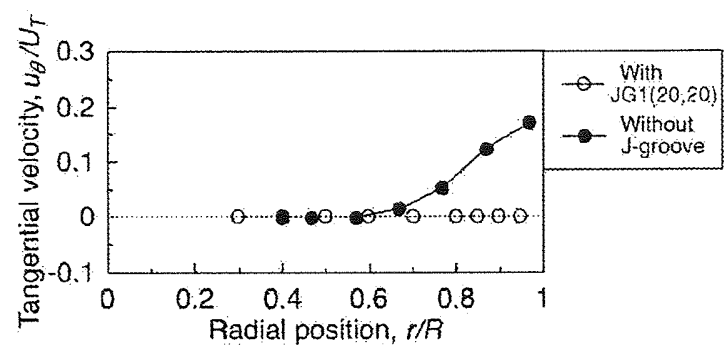

(b) Tangential velocity

Fig.5 Velocity distributions at the inlet of the inducer $(z / D=-0.294)$ in non-cavitating condition. $\phi=0.078,2000 \mathrm{rpm}$.

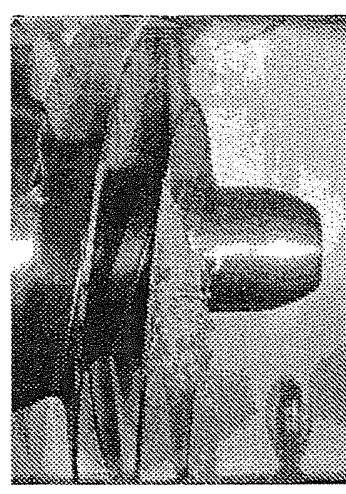

(a) Without J-groove

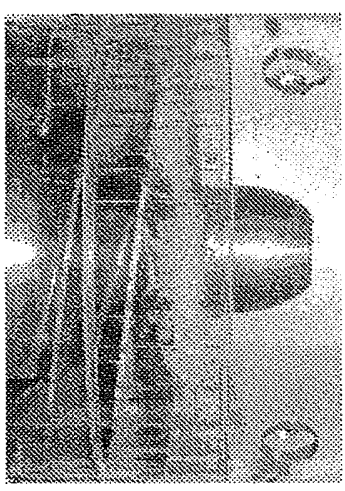

(b) With JG1 $(20,20)$
Fig. 6 Tip cavitation in the inducer without and with JG1 $(20,20)$. $\phi=0.078, \sigma=0.120,3000 \mathrm{rpm}$.

the cavitation surge can be avoided by extending the groove upstream.

Figure 7 shows the cross-section of a new casing with J-groove with the leading edge extended upstream by $20 \mathrm{~mm}$. This groove is called JG2 $(40,20)$. The width, the depth, and the number of J-groove of JG2 $(40,20)$ are the same as those of $\operatorname{JGl}(20,20)$. The occurrence regions of the cavitation instabilities in the case with JG2 $(40,20)$ is shown in Fig. 8 on the suction performance curves. Comparing Fig. 8 with Fig.4(b), the occurrence region of the cavitation surge at higher cavitation numbers and rotating cavitation is decreased in the case with $\mathrm{JG} 2(40,20)$. The asymmetric cavitation was suppressed completely. However, at lower cavitation numbers ( $\sigma \leqq$ 0.04), another type of cavitation surge appeared. 


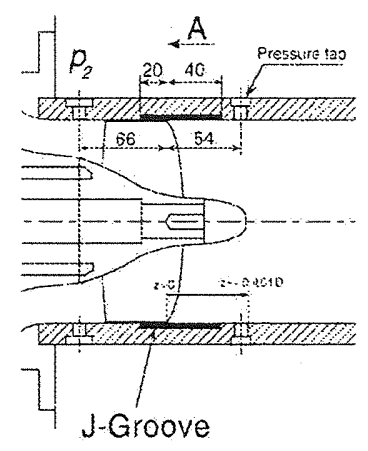

Fig. 7 Cross-section of JG2 $(40,20)$

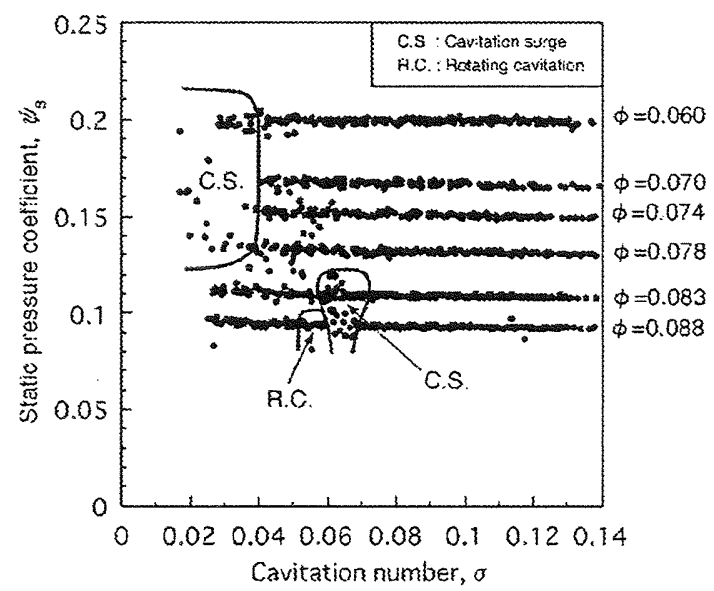

Fig. 8 The occurrence regions of cavitation instabilities on the suction performance curves at various flow coefficients in the case with JG2 $(40,20)$. Rotational speed is 3000 rpm.

Figures 9(a), (b) show photographs of a tip cavitation in the case with JGl $(20,20)$ and JG2 $(40,20)$, respectively, at the lower flow coefficient $(\phi=0.060)$ where the effect of J-groove is remarkable. The cavitation number $\sigma$ is 0.180 . In these figures, it is found that the tip leakage vortex cavitation extends more upstream in the case with JG2 $(40,20)$ as expected. Therefore, the interaction between the tip leakage vortex cavitation and the leading edge of next blade does not occur in the case with JG2 $(40,20)$, and the cavitation surge at higher cavitation number is avoided. For both cases, a ring vortex can be observed at internal radial locations between the leading edges of the blades and the grooves.

Next, we think about the cavitation surge at higher flow coefficients $(\phi=0.083 \sim 0.088)$ and higher cavitation number $(\sigma=0.055 \sim 0.065)$. Figure 10 shows the photographs of tip cavitation under the cavitation surge in the case with $\operatorname{JG} 2(40,20)$ at $\phi=0.088$ and $\sigma=0.065$. The tip leakage vortex cavitation does not extend upstream due to reduced blade loading near the leading edge. The interaction of the tip vortex cavitation with the next blade is clear and this is the reason why the cavitation surge could not be avoided at higher flow rate. Figure 11 shows the spectra of inlet pressure fluctuation in the case with

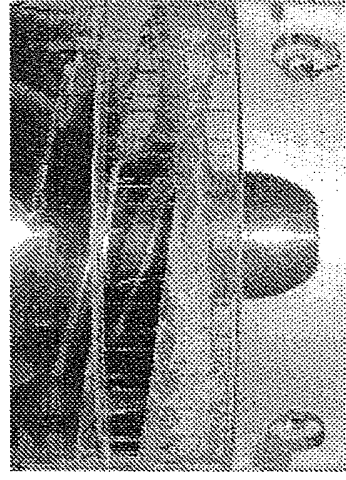

(a) JG1 $(20,20)$

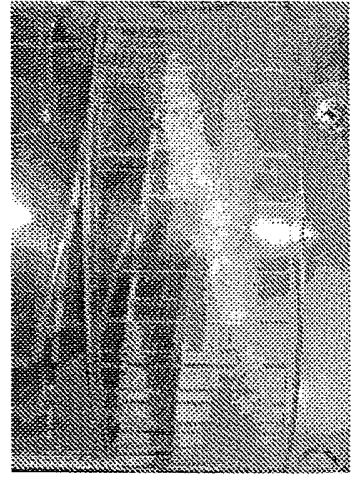

(b) JG2 $(40,20)$
Fig. 9 Tip cavitation in inducer for, $\phi=0.060$, $\sigma=0.180$

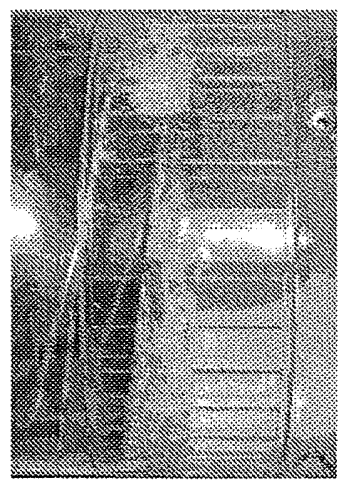

(a) With longer cavity

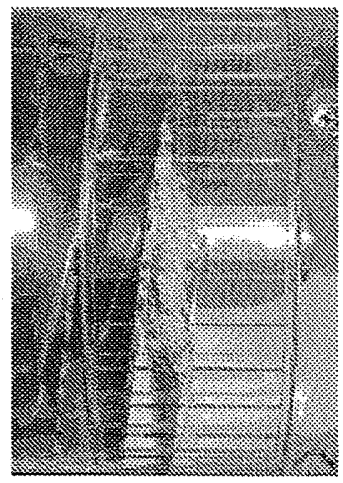

(b) With shorter cavity
Fig.10 Tip cavitation under cavitation surge with JG2 $(40,20)$ for, $\phi=0.088, \sigma=0.065$.

JG2 $(40,20)$ at $\phi=0.088$. The frequency of the cavitation surge at the higher flow coefficients and $\sigma \div 0.060 \sim 0.070$ was about $13 \mathrm{~Hz}$ at $3000 \mathrm{rpm}(50 \mathrm{~Hz})$. The fluctuation of the cavity length in the cavitation surge was also observed in $\operatorname{JGl}(20,20)$ and its frequency is the same as the frequency in JG2 $(40,20)$.

Now we focus on the cavitation surge at the lower flow coefficients $(\phi \leqq 0.078)$ and lower cavitation numbers $(\sigma \leqq 0.040)$. Figure 12 shows a photograph of the tip cavitation just before the occurrence of the cavitation surge at $\phi=0.078, \sigma=0.050$. As shown in Fig.9(b) the interference between the tip leakage vortex cavitation and the leading edge of the next blade can be avoided. However, when the cavitation number decreases, the cavitation between the tip leakage vortex cavitation and the blade surface, which is shown by a red circle in Fig. 12, develops and approaches the leading edge. The cavitation surge shown in Fig. 13 occurs when this tip leakage cavity starts to interact with the leading edge of the next blade. This suggests that the cavitation surge at the lower cavitation numbers ( $\sigma \leqq 0.040$ in Fig. 8 ) is caused by the interference of the leading edge of next blade with the 


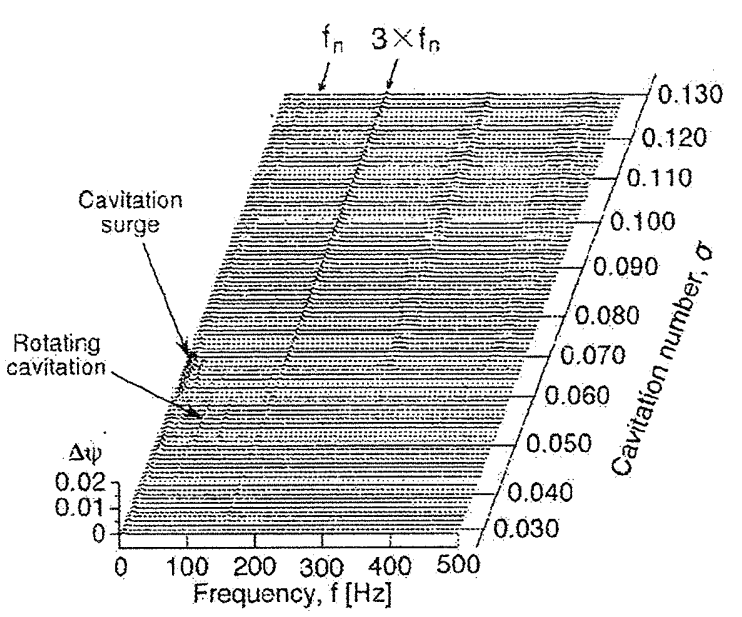

Fig.11 Spectra of inlet pressure fluctuation in the case with JG2 $(40,20) . \phi=0.088,3000 \mathrm{rpm}$.

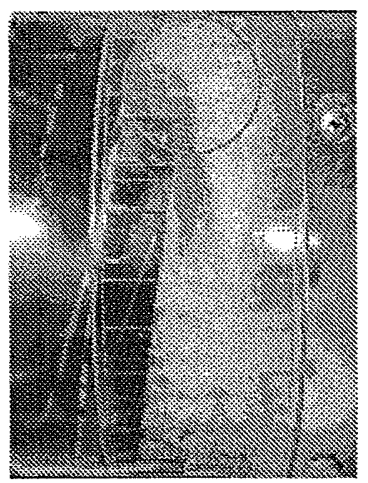

Fig.12 Tip cavitation in the inducer in the case with JG2 $(40,20) . \phi=0.078, \sigma=0.050$.

cavitation between the tip leakage vortex and the blade surface. Figure 14 shows the spectra of the inlet pressure fluctuation at the design flow coefficient $(\phi=0.078)$. The frequency of the cavitation surge at $\sigma \doteqdot 0.030$ is about $5 \mathrm{~Hz}$, which is significantly lower than the frequency $13 \mathrm{~Hz}$ of the cavitation surge at higher cavitation number.

The cavity between the tip leakage vortex cavitation and the blade surface mainly occurs in the grooves, as shown in Figs. 12 and 13. From these results, it is expected that the displacement of the trailing edge of $\mathrm{J}$ groove to the upstream could delay the interference of the cavity with the leading edge of the next blade. Thus, the trailing edge of J-groove was moved upstream by $10 \mathrm{~mm}$. The cross-section of the new casing is shown in Fig. 15 and the groove is called JG3 $(40,10)$. The width, the depth, and the number of grooves of $\mathrm{JG} 3(40,10)$ are the same as those of JGl $(20,20)$ and JG2 $(40,20)$.

Occurrence regions of cavitation instabilities with $J G 3(40,10)$ is shown in Fig. 16. Comparing with Fig. 8 , it is found that the occurrence region of the cavitation surge at lower cavitation numbers is extended. Figure 17 shows photographs of the tip cavitation at $\phi=0.060$ and $\sigma=0.150$.

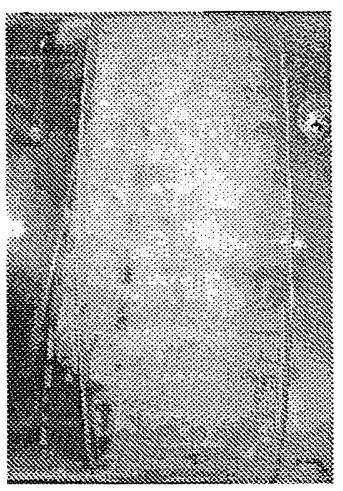

(a) With heavier cavity

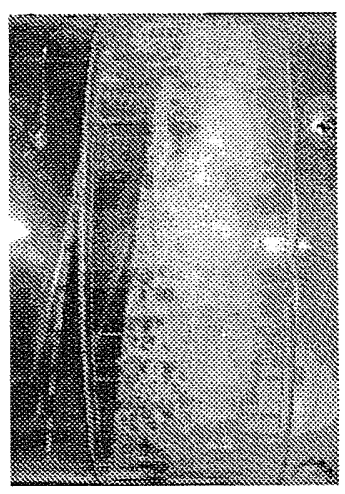

(b) With lighter cavity
Fig. 13 Tip cavitation under the cavitation surge in the case with JG2 $(40,20) . \phi=0.078, \sigma=0.030$.

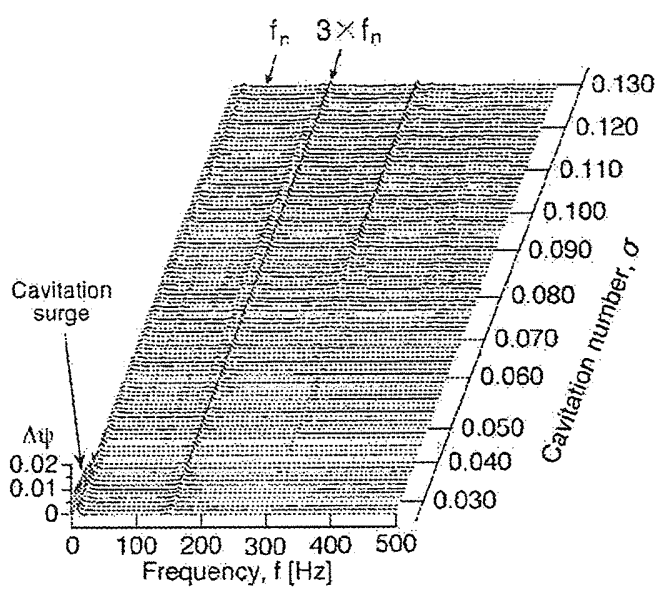

Fig.14 Spectra of inlet pressure fluctuation in the case with JG2 $(40,20) . \phi=0.078,3000 \mathrm{rpm}$.

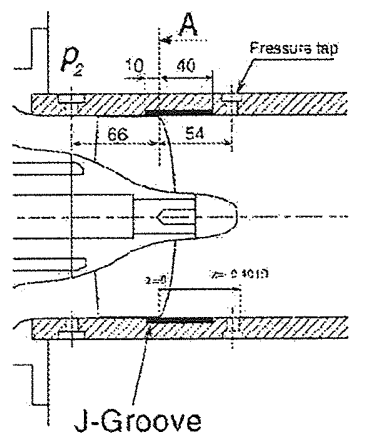

Fig. 15 Cross-section of JG3 $(40,10)$

The angle of the tip leakage vortex cavitation from the blade surface is smaller for the case with JG3 $(40,10)$ as compared with JG2 $(40,20)$ caused by the decrease of the flow rate of backflow in J-groove. Figure 18 shows photograph of the tip cavitation at $\phi=0.078$ and $\sigma=0.050$ just before the occurrence of the cavitation surge. 


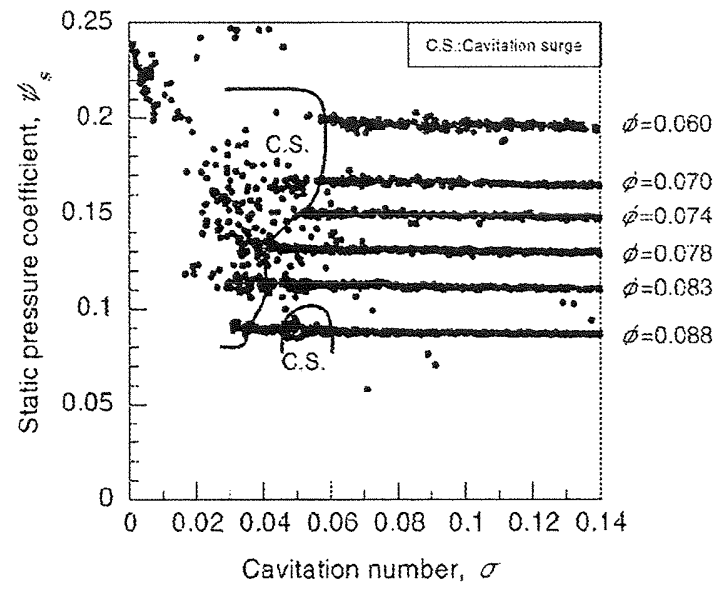

Fig.16 The occurrence regions of cavitation instabilities on the suction performance curves at various flow coefficients in the case with $\operatorname{JG} 3(40,10)$. Rotational speed is $3000 \mathrm{rpm}$.

Comparison with Fig. 12 shows that the cavity is closer to the leading edge for JG3 $(40,10)$.

In an inducer with larger blade angle, the cavitation surge was suppressed completely by increasing the width of J-groove (Kurokawa, J., et. al., 2005). Based on this fact, a new casing with increased groove width $(15 \mathrm{~mm})$ and decreased number (24) was tested. The axial location of J-groove was the same as JG3 $(40,20)$. The occurrence region of the cavitation surge was almost the same as JG3 $(40,10)$.

\section{CONCLUSIONS}

The relation between cavitation instabilities and tip leakage cavitation is discussed by controlling the latter by $\mathrm{J}$-grooves. The results can be summarized as follows

(1) The occurrence region of cavitation instabilities such as the rotating cavitation could be diminished by J-groove.

(2) The cavitation surge in higher cavitation numbers, which was caused by the interference of the tip leakage vortex cavitation with the leading edge of next blade, could be almost suppressed by extending J-groove upstream.

(3) When the cavitation number was decreased, the cavitation surge occurred in lower cavitation numbers in the casings with J-groove extended upstream. This was caused by the interference of the leading edge of next blade with the cavitation between the tip leakage vortex cavitation and the blade surface.

(4) The effect of J-groove is larger at the lower flow coefficients than the design flow coefficient, because the tip leakage vortex cavitation extends upstream and does not interact with the leading edge of next blade.

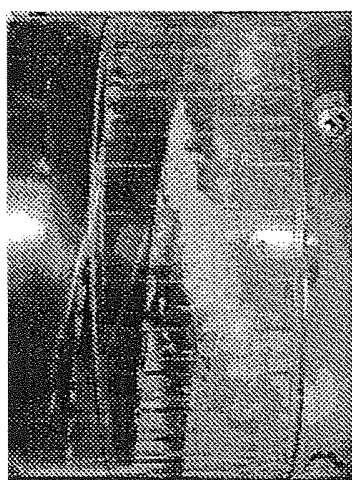

(a) JG2 $(40,20)$

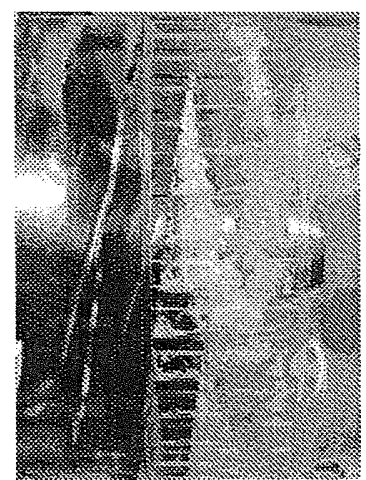

(b) JG3 $(40,10)$
Fig. 17 Tip cavitation in the inducer. $\phi=0.060$, $\sigma=0.150$.

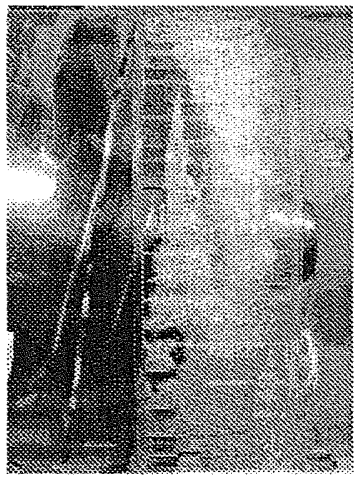

Fig. 18 Tip cavitation in the inducer in the case with JG3 $(40,10) . \phi=0.078 \sigma=0.050$.

(5) At higher flow coefficients, the tip leakage vortex cavitation develops in circumferential direction with or without J-groove and starts to interact with the leading edge of next blade. As a result, the cavitation instabilies are not suppressed at higher flow coefficient.

As mentioned above, it was found that the interference of the tip leakage cavitation with the leading edge of next blade is closely related with the occurrence of cavitation instabilies. By a two-dimensional stability analysis, it has been shown theoretically that the interference of the blade surface cavitation on the blade surface with the next blade causes various modes of instabilies (Horiguchi, H., et. al., 2000). Based on this result, the relation between the cavitation near the tip and cavitation instabilies has been discussed. The results of the present study shows the importance of the tip leakage cavitation and it is suggested that cavitation instability can be suppressed by controlling the tip leakage cavitation, not only by I-groove but also by the design of the impeller. So, we are planning to suppress the cavitation instabilies by changing the design of inducer. 


\section{REFERENCES}

Kamijo, K. et. al., 1993, 'Hydraulic and Mechanical Performance of LE-7 LOX Pump Inducer', Journal of Propulsion and Power, Vol.9, No.6, pp.819-826.

Imamura, H., et. al., 2003, 'Suppression of Cavitating Flow in Inducer by J-Groove', Proceedings of the Fifth International Symposium on Cavitation (CAV2003), Osaka, Japan.

Fujii, A., et. al., submitted, 'Suppression of Rotating Cavitation in an Inducer by J-Groove', (in Japanese), Transactions of the JSME, Series B.

Kurokawa, J., et. al., 2005, 'Suppression of Cavitation in Inducer by J-Groove', (in Japanese), Turbomachinery, Vol.33, No.10, pp.592-600.

Maekawa, Y., 1996, 'Experimental Study of Unsteady Cavitation in an Inducer', (in Japanese), Master's Thesis, Osaka University.

Horiguchi, H., et. al., 2000, 'A Linear Stability Analysis of Cavitation in a Finite Blade Count Impeller', Transactions of the ASME, Journal of Fluids Engineering, Vol.122, No.4, pp.798-805. 